\title{
Virtual Reality-Based Forward Looking Sonar Simulation
}

\author{
Ma Yongzhong \\ Engineering University of Armed Police Force, Xi,an 710086,China \\ myzwjxy@163.com
}

\begin{abstract}
Keywords: Forward-looking sonar simulator; Collision detection; The real time; Obstacle avoidance
\end{abstract} algorithm

\begin{abstract}
In this paper, virtual reality and its related algorithm was used to simulate the installation on the underwater vehicle forward-looking sonar, designs a single beam based on collision detection technique, the mechanical scanning or multi-beam active detection two ways of new type of collision detection analog sound beam, in real time under the premise of guarantee system, sensing complex virtual terrain and mobile obstacles. Software program method is used instead of rotating mechanism such as physical realization simulation of forward looking sonar detection scans, good simulation results have been achieved. Results show that the simulator can provide according to the requirements for the obstacle avoidance system based on real data to construct the information such as the submarine topography and mobile obstacles, to improve the algorithm of obstacle avoidance problem provides a new way of thinking.
\end{abstract}

\section{Preface}

Owing to the power, size and processing unit of AUVs, two major types of Forward Looking Sonar are used: the single-beam scanning sonar and multi-beam sonar system; as there is no essential difference between the single-beam sonar and the multi-beam sonar, multi-beam sonar system is composed of multiple transducer array modules, so it could send and receive multiple beams in working and forms a sector sound transmission area, through which acquire data of the underwater environment[1]. Based on the above principles, two modes of the sonar simulator are designed, including the single-beam and multi-beam scanning.

\section{Simulation Method of Sound Wave}

According to the theory that the acoustic waves through the short-distance can be taken as the ray, using the collision detection segment (VR technique) as a substitute for the sound wave propagation to perceive the virtual environment, is brought forward in the paper[2]. At the same time, a new type of bamboo-shaped detector to reduce the computation and work in the actual resolution of the sonar is devised[3,4], as shown in Fig.1. According to the Fig.1, the collision detection is designed to be bamboo-shaped segment, that is to take the beam transmitted by sonar transducer as the lines, the length of the first detection bamboo segment is the minimum detection distance (its value is $5 \mathrm{~m}$, which means that the smallest resolution is $5 \mathrm{~m}$ ), the bamboo-shaped segment length increases by $5 \mathrm{~m}$ successively, the last cumulative segment length is $200 \mathrm{~m}$ (that is the probing radius of the sonar). Three bamboo-shaped detectors in this Fig. simultaneously place in the horizontal plane perpendicular to AUVs, which could simulate the acoustic beam transmitted by the transducer channel(called to be a collision detection channel) of Forward Looking Sonar in certain direction, and its angle is the vertical angle and its range can be adjusted. Each detection segment could sense the virtual obstacle. When there is the intersection between the segment and the obstacle, there will be the trigger signal given. In addition, this simulation model could not only perceive the obstacle arise within the vision, but could find out the coordinates of the intersection between three beam rays and the obstacle. 


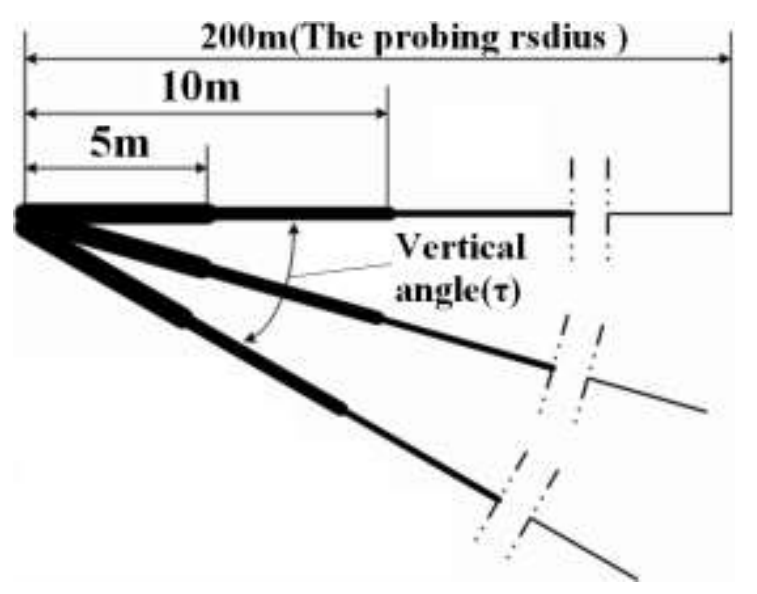

Fig. 1 Sketch map of collision detection segments

The Bamboo-Shaped Collision Detection Segments. In order to facilitate the management, three-dimensional array in form of $\operatorname{seg}[K][J][I]$ is used to define all bamboo-shaped collision detection segment, and $I$ stands for the number of the horizontal beams of sonar, while the values of the single-beam sonar is different from that of the multi-beam sonar, $J$ for the number of vertical beams of sonar, set $J=3, K$ for the number of collision detection segment needed to achieve the sonar probing radius, the calculating formula of $I 、 K$ are as follows:

$$
\begin{aligned}
& I=\eta / \mu \\
& K=\omega / \sigma
\end{aligned}
$$

Among them, $\eta$ stands for the horizontal angle of Forward Looking Sonar, $\mu$ for the horizontal angle between two adjacent transducers of multi-beam Forward Looking Sonar or the stepping angle of single-beam Forward Looking Sonar scanning, $\omega$ for the probing radius of Forward Looking Sonar, $\sigma$ for the smallest resolution of the length of the collision detection segment. The value of $\omega$ to be $200 \mathrm{~m}$ and $\sigma$ to be $5 \mathrm{~m}$.

Location of the collision detection segments.After the establishment of a bamboo-like collision detection segments, its location needs to be defined which includes the initial location of bamboo-shaped collision detection segments and relative positions among them. The structure of the detection channel of single-beam Forward Looking Sonar is the same as that of the multi-beam sonar (Fig.1), while they only differ in the angles[5]. The multi-beam Forward Looking Sonar is illustrated by serving as an example in this paper. Fig. 2 shows the sound propagation characteristics of the multi-beam Forward Looking Sonar.

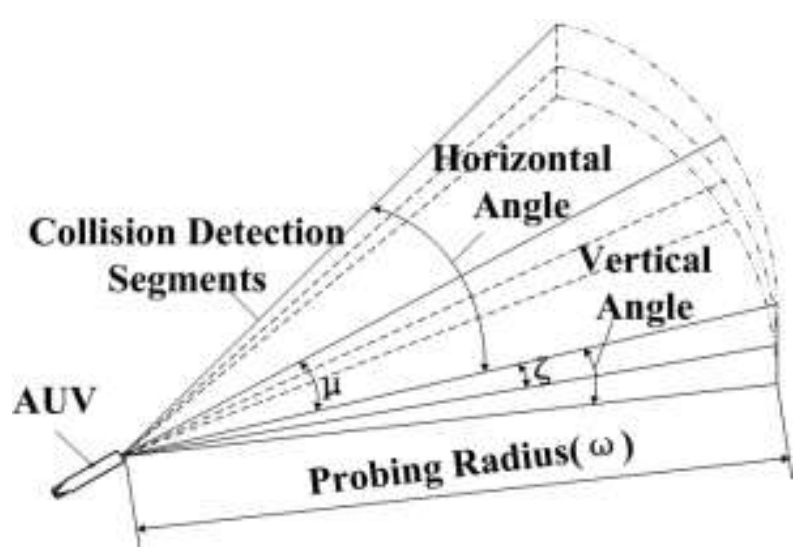

Fig. 2 Sonar beam pattern

It is essential to accurately place each collision detection segment. Supposed that the Forward Looking Sonar is installed in the bow of AUV, the starting points of all collision detection segments are 
in the bow of AUV. The relative position among collision detection segments can be respectively written as,

$$
\begin{aligned}
& \left\{\begin{array}{l}
x[k][j][i]=\cos \left(\frac{1}{2} \tau-\mu \times j\right) \cos \left(\frac{1}{2} \eta-\xi \times i\right) \\
y[k][j][i]=\cos \left(\frac{1}{2} \tau-\mu \times j\right) \sin \left(\frac{1}{2} \eta-\xi \times i\right) \\
z[k][j][i]=\sin \left(\frac{1}{2} \tau-\mu \times j\right)
\end{array}\right. \\
& \operatorname{len}[k]=5+5 k
\end{aligned}
$$

Where $x[k][j][i], y[k][j][i]$ and $z[k][j][i]$ are the coordinates of the unit vector of collision detection segment $\operatorname{seg}[k][j][i]$ in the detecting coordinates system of the Forward Looking Sonar, len $[k]$ is the length of $\operatorname{seg}[k][j][i], \tau$ stands for the vertical angle of the Forward Looking Sonar, $\eta$ for the horizontal angle of Forward Looking Sonar, $\mu$ for the horizontal angle between two adjacent transducers of multi-beam Forward Looking Sonar or the stepping angle of single-beam Forward Looking Sonar, $\xi$ for the vertical spatial angle between two adjacent collision detection, (Fig. 2). $I, j, k$ is the signs for the collision detection segment corresponding to the elements of seg, where $i \in\{0,1, \cdots I-1\}, j \in\{0,1,2\}$, and $k \in\{0,1, \cdots, K-1\}$.

\section{Scanning Simulation and Data Record}

Forward-looking sonar data recording format. In this paper, 2D grid icon description is used to express the local environment information detected by sonar detector, which is due to that the intelligent body of the underwater vehicle is with limited capability to process the information and the grid icon description is with little calculation, therefore, it could meet the requirement of obstacle avoidance system. Schematic diagram of the grid method is shown in Fig. 3. 2D rectangular grid is used in the grid coordinate system to describe the obstacle information, each rectangular grid has a cumulative $\mathrm{CV}$ value ( $\mathrm{CV}$ value is the possibility of the existence of obstacle after the sensor information fusion), while the selected grid size has a direct impact on the performance of path planning algorithm[6].

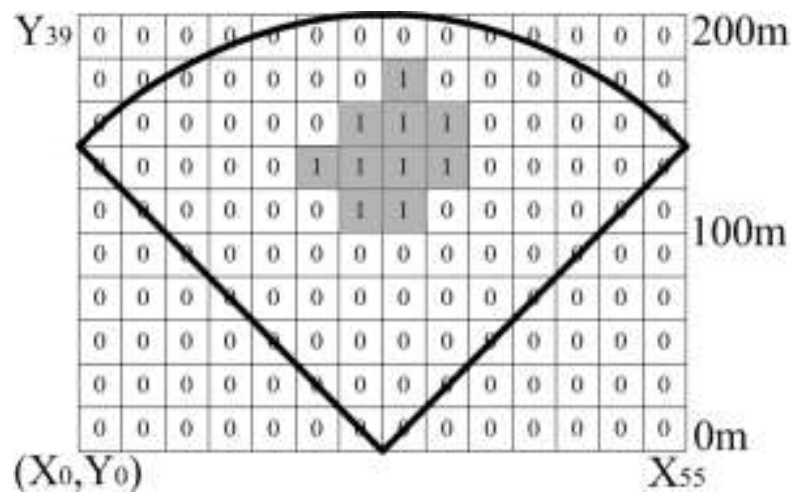

Fig. 3 Sketch Map of obstacle in 2D grid recording

Scanning Simulation and Data Recording Method of Single-beam Forward Looking Sonar. To simulate the beam scanning of the single-beam Forward Looking Sonar, a collision detection channel perpendicular to the craft's horizontal plane is used to simulate the sonar beam width sent by single-bema Forward Looking Sonar transducer, then activates or turns off adjacent collision detection channels to simulate the sonar scanning probe mechanism, each time experiences the whole probing range which is a tempi of sonar probing[7]. 
Multi-beam swath-type exploration model and data logging. To simulate the multi-beam Forward Looking Sonar, activate or turn off all the collision detection channels according to the probing cycle of Forward Looking Sonar to simulate the scanning of multi-beam Forward Looking Sonar.

\section{Experiment}

Select an $11000 \mathrm{~m} \times 8500 \mathrm{~m}$ landscape model from the seabed topography database, based on contour DEM data[8], in use of the Polymesh rectangular grid generation, its topographical features include the sea walls, trenches and ridges[9]. The simulation result of the Forward Looking Sonar is shown in Fig. 4,Left is the diagram of the single-beam Forward Looking Sonar simulation, while the right for the multi-beam Forward Looking Sonar simulation. The collision detection channel in green stands for the trigger, that is the obstacle has been detected (seafloor topography), while the red for that it is not triggered, which shows that there is no any obstacle detected.
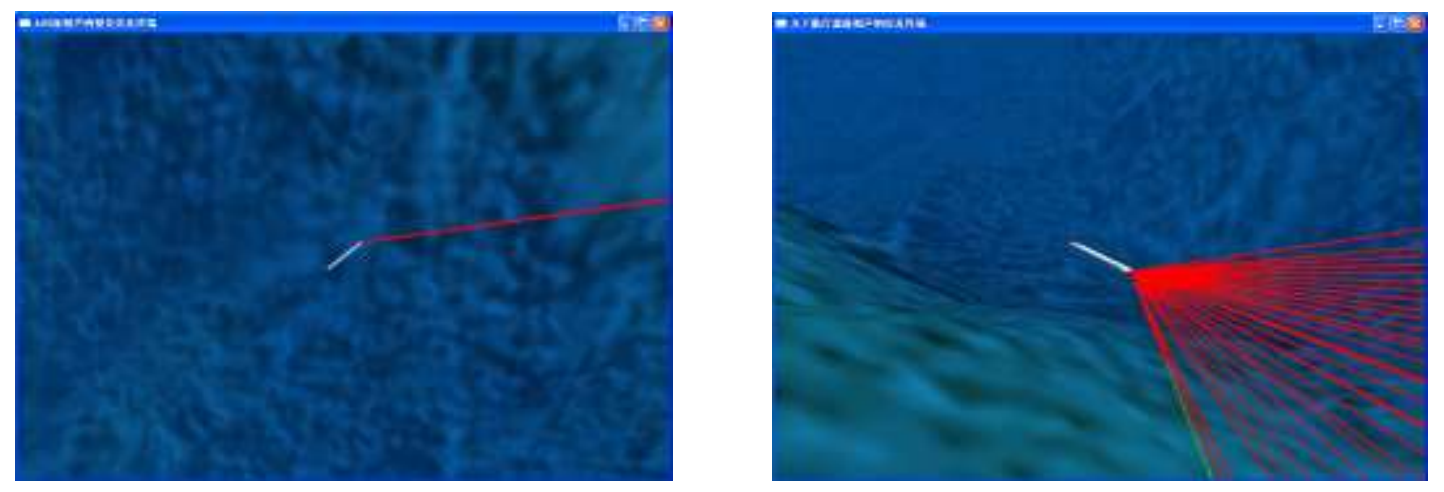

Fig. 4 Simulated images of two types of Forward Looking Sonar simulators

(left: single-beam and right: multi-beam)

Simulator transmits the data in use of the sonar scanning cycle frequency and could write the matrix stack data into the TXT format document[10], the real-time record of multi-beam sonar detection data is shown in Fig. 5(the simulation data generated from the single and multi-beam sonar is with the identical format) [11]. The system sensor simulation data is transmitted according to the actual transmission format and transmission frequency via the serial port, and its purpose is to better simulate the transmission characteristics of the sensor and represent the process of sonar data transmitting to the navigation and control embedded processor; its implementation is as follows: first is to get the sonar data packets and then transmits it to the underwater vehicle's navigation and control processor in the way of serial communication.

\section{Conclusion}

In this paper, the authors devise a new type of collision detector to simulate the sound waves in use of the virtual reality and relevant algorithm based on the collision detection technology. This kind of sonar, in the premise of ensuring the real-time nature of system, could perceive the complex virtual terrain, and it is the most close to the actual sonar imaging in use of the software programs in lieu of the physicals such as rotating bodies to simulate the Forward Looking Sonar detection and scanning, which achieves a good simulation result.

The simulator could provide the marine terrain and movement obstacles based on the actual data according to the obstacle avoidance requirement and provide a new idea for improving the obstacle avoidance algorithm. 


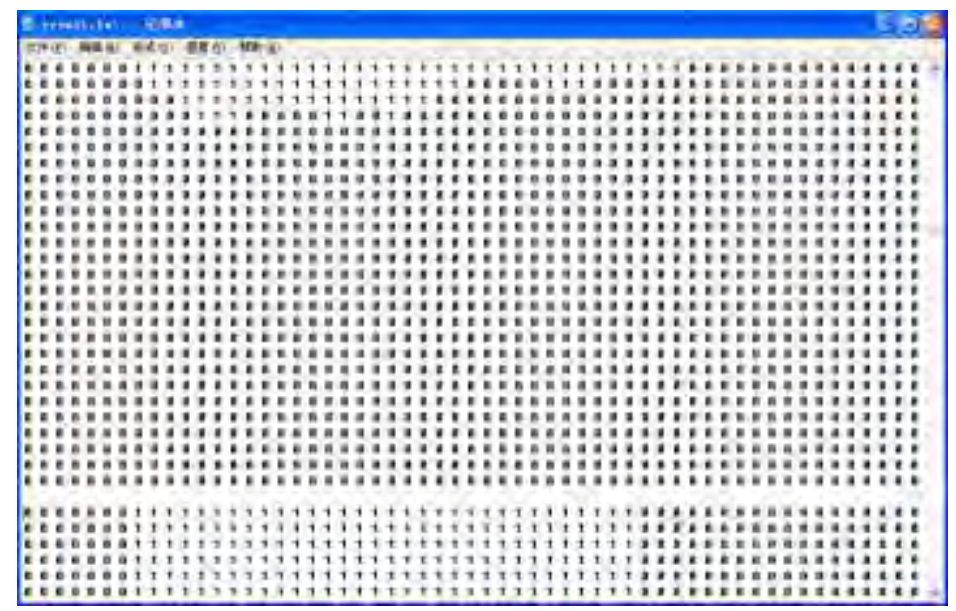

Fig. 5 Simulation Data of Multi-Beam Sonar (two successive tempi)

\section{References}

[1] Wang Yi-Tong.Forward-looking scan sonar imaging and target feature extraction [D]. Qingdao: China ocean university, 2009.

[2] Wang Cheng,Li Li-Jun,Zhou Jun-Qing.V ega real time three dimension visual simulation technology [Ml. Wuhan: huazhong university of science and technology press, 2005.

[3] Wang Cheng,Zhou Jun-Qing,Li Li-Jun.C rector insists the visual simulation modeling technology [M]. Wuhan: huazhong university of science and technology press, 2005.

[4] Kenneth D. Harris, Michael Recce. Experimental Modeling of Time-of-Flight Sonar. Robotics and Autonomous Systems 24 (1998) 33-42.

[5] Bo-Chang Chen, Jung-Hua Chou. A Jump-U model of echo pattern for a sonar ranging module. Applied Acoustics 69 (2008) 1299-1307.

[6] Philip Bouxsein, Edgar An. A Sonar Simulation used to develop an Obstacle Avoidance System. OCEANS 2006 - Asia Pacific. 16-19, pp. 1-7, 2007.

[7] J. Raezkowsky. Simulation of Cameras in Robot Applieation. IEEE JCGA1989, 9(1):16-25.

[8] J.M. Bell, L.M.Linnett. Simulation and analysis of synthetic sidescan sonar images. Radar, Sonur Navig. Vol 144, No 4, pp. 219-226, 1997.

[9] Le-Diem Bui, Yong-Gi Kim. An obstacle-avoidance technique for autonomous underwater vehicles based on BK-products of fuzzy relation. Fuzzy Sets and Systems 157 (2006) 560-577.

[10] Peter Mossinger, Daniel Polani. A virtual testbed for analysis and design of sensorimotoric aspects of agent control. Simulation Practice and Theory 5 (1997) 671-687.

[11] U. Castellani, A. Fusiello. A complete system for on-line 3D modeling from acoustic images. Signal Processing: Image Communication 20 (2005) 832-852. 